\title{
La empleabilidad de las fuerzas armadas por los Estados en una nueva época. El caso peruano ${ }^{1}$
}

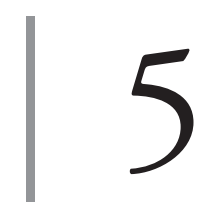

https://doi.org/10.21830/9789585377141.05

\author{
Manuel Alexis Bermúdez Tapia ${ }^{2}$ \\ Universidad Privada San Juan Bautista \\ Paola Alexandra Sierra-Zamora ${ }^{3}$ \\ Escuela Militar de Cadetes "General José María Córdova"
}

\section{Resumen}

El objetivo principal del capítulo es analizar la empleabilidad de las fuerzas armadas por los Estados en actividades de apoyo social ante catástrofes ambientales o emergencias sanitarias, como la ocasionada por la pandemia de Covid-19; actividades de apoyo a la defensa de recursos e intereses prioritarios de los países, como la protección de los recursos marítimos, la defensa de la gobernabilidad y el control migratorio, con especial énfasis en el caso peruano. Con este propósito se empleó una metodología deductiva, con un enfoque sistémico-descriptivo y analítico. Se concluye que las fuerzas militares en Latinoamérica han tenido que ejecutar nuevas funciones para responder efectivamente a la emergencia de una nueva geopolítica internacional, la cual ha hecho necesario que los Estados realicen modificaciones estructurales en la administración de sus recursos nacionales para garantizar la seguridad y defensa de la nación.

Palabras clave: Estados; Fuerzas Armadas; Perú; crisis sanitarias.

1 Este capítulo presenta los resultados colaborativos de dos proyectos de investigación: (1) "Ética militar en entornos complejos de seguridad y defensa: lecturas y aportes desde la experiencia de las Fuerzas Militares de Colombia”, del grupo de investigación Masa Crítica, de la Escuela Superior de Guerra "General Rafael Reyes Prieto", Colombia, categorizado en B por Minciencias y con código de registro COL0123247, y (2) "Análisis de la institucionalidad democrática en el Perú”, de la Facultad de Derecho de la Universidad Privada San Juan Bautista, aprobado por Resolución de Vicerrectorado de Investigación N. - 2019-061VRI-UPSJB. Los puntos de vista pertenecen a los autores y no reflejan necesariamente los de las instituciones participantes.

2 PhD y magíster en Derecho de la Pontificia Universidad Católica de Argentina. Abogado Summa Cum Laude de la Pontificia Universidad Católica del Perú. Profesor investigador de la Universidad Privada San Juan Bautista y profesor de la Facultad de Derecho de la Universidad Nacional Mayor de San Marcos, Perú. Orcid: http://orcid.org/0000-0003-1576-9464 - Contacto: manuel.bermudez@upsjb.edu.pe

3 PhD y magíster en Derechos Humanos, Democracia y Justicia Internacional de Universitat de València, España. Abogada de la Universidad Católica de Colombia. Investigadora del grupo de investigación Ciencias Militares, de la Escuela Militar de Cadetes "General José María Córdova”. OrCid: https:// orcid.org/0000-0002-3146-7418 - Contacto: paola.sierra@esmic.edu.co 


\section{Introducción}

Durante el año 2020 se registraron cuatro hechos importantes en Perú, donde las Fuerzas Armadas fueron evaluadas por una triple exigencia que las obligó a desarrollar una posición institucional con respecto de cada desafío socio-político-económico y sanitario en ciernes, debido a las condiciones de ingobernabilidad y crisis severa.

La gravedad de cada situación identificó características particulares y específicas que permiten evaluar la empleabilidad de las Fuerzas Armadas peruanas de una forma superior a su uso habitual, superando:

1. Los enfrentamientos bélicos con Ecuador (1941, 1981 y 1995).

2. El periodo del terrorismo (1980-2000).

3. Las situaciones de desastres naturales durante los últimos ańos (1982, 1997, 2014 y 2017).

La evaluación del papel institucional y pro gobernabilidad ejecutado por las Fuerzas Armadas peruanas durante el periodo comprendido entre la disolución del Congreso (30/09/2019) y la crisis de gobernabilidad del mes de noviembre del 2020 ha constituido la principal referencia en la cual el Estado peruano ha registrado una participación cívica y democrática del Ejército peruano, la Marina de Guerra y la Fuerza Aérea del Perú sin precedentes en la historia del país.

Complementariamente, el apoyo y control de la población ante la crisis sanitaria provocada por la pandemia de la Covid-19 y el control de fronteras durante la pandemia constituyen los elementos más referenciales a favor de la población. Finalmente, las acciones de defensa preventiva de los recursos biológicos marinos ante la incursión de buques chinos a finales del 2020 permiten identificar cuatro situaciones en las cuales las Fuerzas Armadas peruanas han participado en beneficio del país.

Cuatro referencias que permiten evaluar la importancia que tienen las fuerzas militares en un país en épocas contemporáneas, especialmente cuando no se registran conflictos militares significativos, como sucede en América Latina (De Pieri, 2013, p. 10), donde el contexto militar tradicional tran- 
sita hacia un nuevo escenario (Álvarez \& Duque, 2020). Este nuevo rumbo permite proyectar una condición de empleabilidad de las fuerzas militares internas para atender nuevos problemas que afectan la seguridad y la defensa nacional, en particular porque las acciones de interdicción y lucha contra el narcotráfico, la defensa de los recursos naturales y la ciberseguridad exigen una mayor presencia de instituciones castrenses en la ejecución de políticas públicas, que requieren una profunda formación en ética militar (García, 2019; Guerrero, 2019; Hurtado \& Doria, 2020).

El enfoque metodológico empleado permite evaluar el ámbito de empleabilidad de las fuerzas militares en los países sudamericanos mediante un estudio cualitativo, descriptivo y causal, basado en el análisis documentario y la evaluación de un caso en particular, el cual se limita a la realidad de Perú en un periodo específico: desde octubre del 2019 hasta diciembre de 2020, en particular porque la realidad sociopolítica del país fue severamente afectada y el papel de las Fuerzas Armadas peruanas fue impecable en la defensa de la democracia, la gobernabilidad, la seguridad y el control de la población.

De este modo, es posible apreciar el contexto de adaptación de las políticas de seguridad y defensa nacional en la región latinoamericana por parte de los Estados latinoamericanos, que ante la insuficiencia de recursos y ante la presión provocada por el surgimiento de nuevas exigencias se ven en la necesidad de adaptar las acciones y decisiones éticas de sus fuerzas militares para garantizar su soberanía y seguridad nacional, así como proteger sus recursos (Moreno, 2014).

\section{El cambio de perspectivas y el surgimiento de nuevos elementos de presión a los Estados}

Un análisis del periodo que corresponde al final de la Guerra Fría (Morote, 1999, p. 15) y el inicio del nuevo milenio (Chan, 2005, p. 28) permite observar un proceso de transformación general y sustancial, que ha provocado un nuevo escenario en el ámbito de las relaciones internacionales (Cujabante, 2019).

El nivel de antagonismo de dos bloques ideológicos, políticos, internacionales, económicos y comerciales generado por los Estados Unidos de América y la ex-Unión de Repúblicas Socialistas Soviéticas ha dado paso a un mundo 
mucho más globalizado (Bermúdez-Tapia, 2020a, p. 292), con una mayor presencia y empleo de tecnología digital basado en el uso de medios audiovisuales, en el cual además prevalece un sistema capitalista esquematizado en tres características impuestas por

1. Los Estados Unidos de América (modelo tradicional).

2. China (modelo vinculado al ámbito de las inversiones económicas).

3. Rusia (respecto del modelo político-internacional sobre la base de una posición expectante bajo el amparo de un poderío militar).

Un panorama internacional novedoso que permite identificar algunas características representativas:

1. La reducción de guerras internacionales entre países (Gatt, 2013), sea por motivos de reclamación o ampliación de territorios.

Inclusive situaciones de antagonismo tradicional como las registradas en las fronteras de China con India o entre Pakistán con India, no han provocado situaciones bélicas referenciales significativas (Vasapollo et al., 2007, p. 118).

En el mismo sentido, desde el inicio del nuevo milenio no se ha registrado ninguna guerra, con el modelo tradicional, en el cual se pudiera haber registrado la participación de las fuerzas militares de los países enfrentados.

2. La generación de un contexto internacional que recurre en forma secundaria a la presión militar, especialmente porque las sanciones o vetos políticos-económicos derivados de acciones de carácter político-internacional resultan mucho más significativos en el escenario internacional.

La imposición de embargos económicos (sanciones) a países contrarios a la línea de acción liderada por los Estados Unidos o la Unión Europea, como las registradas contra:

a. Irán por la ejecución y el desarrollo de un programa nuclear sin fiscalización o autorización. 
b. Venezuela, especialmente por el contexto político generado por el sistema económico y político promovido por el gobierno de Nicolás Maduro, que sigue el modelo impuesto por Hugo Chávez.

c. Rusia, especialmente por las acciones de anexión de territorios de Crimea y Sebastopol en el 2014.

Esto permite apreciar una política específica por parte de los Estados Unidos de América que resulta mucho más significativa y efectiva que una incursión militar (Narváez \& Rodríguez, 2005).

Otra referencia paralela a lo que se ha detallado es que las acciones militares y económicas ejecutadas por algunos países, como los Estados Unidos de América, resultan superiores a las decisiones que impone las Naciones Unidas, pese a que esta entidad es la única con legitimidad para imponer tales medidas.

3. El surgimiento de nuevas exigencias a los Estados que ha generado la necesidad de una adaptación a nuevos modelos de desarrollo de políticas de seguridad y defensa nacional (Griffiths, 2011), en particular porque el "enemigo" ya no es un país sino una organización vinculada a la ejecución de actividades ilícitas. Se trata de organizaciones vinculadas a los siguientes ámbitos:

a. Terrorismo en Perú (1980-2020) y guerrillas en el contexto del conflicto armado interno, tal como sucedió en Colombia conforme detallan Bermúdez-Tapia, Sierra-Zamora y Fernández Osorio (2020, p. 85).

b. Narcotráfico (Fernández, 2020).

c. Ejecución de actividades de minería ilegal o depredación de recursos naturales.

d. Las que desarrollan actividades de lavado de activos o delitos de carácter internacional están predeterminando un nuevo ámbito de evaluación y los Estados están adaptándose a estas exigencias (Basombrío et al., 2006, p. 25).

4. La importancia del uso de las fuerzas militares en la ejecución de acciones de defensa de recursos naturales, minerales, ambientales y 
acuíferos (Welzer \& Obermeier, 2010, p. 283) porque estos se están convirtiendo en el nuevo foco de atención de futuros enfrentamientos entre los países, en particular por las implicaciones económicas, sociales y políticas que puedan tener.

Téngase en cuenta en este punto que las proyecciones prevén conflictos armados por el acceso a los siguientes recursos:

a. Fuentes de agua (Bruzzone, 2008, p. 31).

b. El uso de materiales y recursos minerales para el suministro de energía (García \& Ronquillo, 2005, p. 38).

c. El acceso a fuentes de provisión de recursos biológicos (Tansey \& Rajotte, 2009, p. 56).

Por tanto, surge la necesidad de configurar nuevas políticas de Estado y políticas públicas vinculadas al ámbito de la empleabilidad de las fuerzas militares, porque las condiciones tradicionales para su operatividad se han modificado (Pastrana \& Gehring, 2018). Específicamente, se registran los siguientes elementos más significativos:

1. La necesidad de una adaptación de equipos y material bélico a nuevas exigencias. El uso de nuevas tecnologías, como también en cuanto a la capacidad de operatividad en contextos contemporáneos, en los cuales el enemigo no es un país sino personas y organizaciones que pueden estar bajo una condición delictiva o que pueden operar en forma informal y sin una organización específica, pero que pueden generar una condición negativa a los intereses de un país (Cañedo, 2014, p. 224).

2. La reducción de material bélico. En el más amplio sentido del término, porque el uso de una mejor tecnología permite una mejor empleabilidad de equipos sin mucho personal militar, con lo cual se salvaguarda la integridad y las vidas de los militares, como también porque ello implica una reducción de costos significativos (Shaw, 2005, p. 53).

El uso de tecnología satelital, de drones, de equipos bélicos de vigilancia y la recurrencia al uso de equipos informáticos para la 
ejecución de actividades de interdicción, intervención y fiscalización (Roland, 2016) es representativo en la mayoría de las fuerzas armadas en la región, especialmente las que disponen de un mayor manejo de recursos financieros y presupuestarios, tal como ocurre en Argentina, Brasil, Chile y Colombia (Infodron.es, 2020).

3. La exigencia de mayores recursos financieros y presupuestarios para las fuerzas militares. En este punto se debe detallar que los países latinoamericanos no disponen de una autonomía suficiente para la generación de dicha tecnología o para el mantenimiento autónomo de dichas actividades (Fukuyama, 2008).

La dependencia de tecnología ha condicionado a las fuerzas militares latinoamericanas y prueba de ello es que la gran mayoría de países no ha generado ni producido material bélico significativo de forma autóctona. Inclusive el nivel de operatividad de sus fuerzas armadas se ha reducido porque los cambios de prioridades resultan notorios.

En otra perspectiva, un significativo grupo de países emplea a sus fuerzas armadas para establecer las garantías y condiciones de sostenibilidad de sus propios gobiernos, especialmente porque se enfrentan a conflictos internos, por problemas étnicos o guerras civiles (Trebilcock \& Mota, 2011, p. 134).

4. Complementariamente al último punto, surge una nueva condición negativa contemporánea derivada de los altos costos económicos y presupuestarios que se derivan de actividades de naturaleza militar: la fuga de recursos humanos al sector privado.

Chile ha reconocido que anualmente pierde pilotos en sus fuerzas aéreas porque estos optan por ingresar a la actividad comercial aeronáutica, sin considerar el elevado presupuesto que sus gobiernos han empleado para su formación, capacitación y especialización (Infodefensa, 2020).

Estos elementos condicionan y predeterminan las políticas que los países deben establecer en función de una proyección temporal y ejecución 
de acciones específicas, con lo cual es posible afirmar que estamos ante un nuevo periodo de evaluación en donde las fuerzas militares asumirán nuevas funciones.

\section{Las fuerzas militares asumen nuevos roles}

Tomando en cuenta el contexto latinoamericano, es posible identificar dos ejes de acción en los cuales las fuerzas armadas se han adaptado a las exigencias que han incidido en sus países.

\section{Las actividades de apoyo social ante catástrofes ambientales}

América Latina es una región cuya geografía y condiciones morfológicas resultan ser muy extensas y complicadas de administrar y de fiscalizar. En este sentido, es posible identificar algunas regiones muy particulares, especialmente porque en casos de desastres naturales han sido las fuerzas militares las que han participado de modo activo en las actividades de atención de emergencias humanitarias, de apoyo social y de atención de necesidades de la población afectada (Giraldo, 2019). Regiones como:

1. La región amazónica, que registra siete millones y medio de kilómetros cuadrados. En esta zona Brasil, Venezuela, Colombia, Ecuador, Perú, Bolivia, Paraguay y las tres Guayanas registran una frontera común (CEPAL, 2020).

Un territorio que inclusive no es explorado en su totalidad y que registra espacios en los cuales la presencia humana no es permanente o sostenida debido al clima, la presencia de animales parasitarios y dificultades de acceso.

Sin embargo, es la región que reporta dos elementos materiales que serán ejes de atención especial y donde las fuerzas militares de los países latinoamericanos deberán adaptarse (Varea, 1995, p. 236) para proteger el ecosistema y los recursos hídricos, en particular porque el río Amazonas registra la mayor concentración de 
agua dulce en el planeta y dicho recurso ya se reporta como un bien evaluado con los parámetros del sistema financiero y bursátil.

2. Los Andes, que representa la columna vertebral de Sudamérica, con un largo total de $7.000 \mathrm{~km}$, con zonas con un ancho que pueden registrar 160 kilómetros (Perú, Bolivia), ubicado entre Colombia, Ecuador, Perú, Bolivia, Chile y Argentina (Dollfus, 2014). En esta extensión territorial se debe apreciar:

a. Zonas alto andinas donde se registra la presencia de comunidades, sobre todo indígenas, que en épocas de catástrofes naturales son evacuadas por las fuerzas militares. En este sentido, cuando ocurren terremotos, heladas, erupciones volcánicas, desprendimiento de tierras en las montañas o emergencias sociales vinculadas al ámbito de un enfrentamiento con actividades de exploración o explotación de hidrocarburos, las fuerzas militares son las únicas entidades estatales que hacen presencia permanente en dichos ámbitos geográficos.

b. Zonas de accesibilidad limitada donde se han registrado situaciones excepcionales provocadas por personas que no han podido superar las dificultades propias de la geografía, como reportes de personas desaparecidas en incursos o exploración en glaciares o cadenas montañosas elevadas o el registro de accidentes vinculados al ámbito del transporte de personas.

3. Regiones hidrográficas como el Orinoco o el Paraná, que constituyen ámbitos geográficos particulares y diferenciados de la realidad amazónica.

4. El océano que rodea al zócalo continental, en particular porque representa una región en la cual se evalúa la protección de los recursos biológicos y minerales ubicados tanto en el océano Pacífico como en el océano Atlántico.

En este ámbito, obsérvese las acciones ejecutadas por el gobierno argentino, que añadió $1^{\prime} 782.000 \mathrm{~km}^{2}$ a su territorio, sobre la base de una interpretación vinculada a las características morfológicas de la plataforma continental. Esta acción fue aprobada por la Comisión 
de Límites de la Plataforma Continental de las Naciones Unidas, derivado de la interpretación y aplicación de la Convención sobre Derechos del Mar (CONVEMAR) en el año 2016 (Cancillería, 2016). Complementariamente, la defensa de los límites marítimos no ha sido un asunto de fácil evaluación en el ámbito de las relaciones diplomáticas entre los países de la región (Velázquez, 2014), donde se han registrado:

a. La dispuesta territorial entre Colombia y Nicaragua, definida por la Corte Internacional de Justicia de La Haya en el 2012 (Alvarado, 2014, p. 241).

b La disputa territorial entre Perú y Chile, definida por la Corte Internacional de Justicia de La Haya en el 2014, que implicó el establecimiento de un nuevo límite fronterizo entre los dos países después de la Guerra del Pacífico en 1879 (López, 2014, p. 1.133).

\section{La ejecución de actividades de apoyo a la defensa de recursos e intereses prioritarios de los países: el caso peruano}

En particular, para la atención de nuevas necesidades, como:

1. La protección de los recursos biológicos y ecosistemas amazónicos, andinos y marítimos en la mayor parte del territorio latinoamericano.

2. La ejecución de actividades de control migratorio, especialmente luego de la crisis de Venezuela.

3. Las acciones de lucha contra el narcotráfico y el tráfico de productos derivados de cultivos ilícitos (Sierra-Zamora et al., 2020, p. 19), la cual fue regulada por la Ley 30796 y que provocó la evaluación de su constitucionalidad, cuando el poder ejecutivo planteó su inconstitucionalidad ante el Tribunal Constitucional, lo cual generó la sentencia recaída en el Expediente N.o 0011-2019-PI (Tribunal Constitucional, 2020).

Inexplicablemente, el poder ejecutivo peruano cuestionó una norma que le permitía un mejor margen de acciones legales y de acción 
en los escenarios geográficos donde se habían declarado estados de emergencia, a lo cual el Tribunal Constitucional validó la posición del Congreso de la República al interpretar en forma orgánica los artículos $8 .^{\circ}, 43 .^{\circ}, 118 .^{\circ}, 137 .^{\circ}, 164 .^{\circ}, 165 .^{\circ}, 166 .^{\circ}$ y $167 . .^{\circ}$ de la Constitución Política de 1993 (Bermúdez-Tapia, 2008), que reproducimos en la parte final del documento, en los Anexos.

Con la evaluación positiva en las actividades de control y represión del Tráfico Ilícito de Drogas, las Fuerzas Armadas peruanas adquieren una condición adicional a sus funciones que se desprenden de la política de Estado sobre seguridad y defensa nacional, regulado por Decreto Supremo N. o 012-2017-DE (Gobierno del Perú, 2017).

\section{Las Fuerzas Militares peruanas y el cambio de funciones durante los últimos años}

Las Fuerzas Armadas peruanas, luego de los gobiernos de Juan Velasco Alvarado (1968-1975), Francisco Morales Bermúdez (1975-1980) y Fernando Belaúnde Terry (1980-1985) han registrado una crisis significativa, tanto operativa como institucional, especialmente porque se han visto condicionados por situaciones de corrupción y negligencia, en particular durante:

1. El gobierno de Alan García Pérez durante los años 1985-1900 y 2006-2011. El nivel de perjuicio provocado a las Fuerzas Armadas ha sido expuesto en múltiples situaciones, pero no se ha registrado alguna situación judicial o política en contra. Los hechos más significativos en contra de las Fuerzas Armadas fueron:

a. El desistimiento en la compra de catorce aviones Mirage 2000 y la ejecución de una transferencia ilegal de los mencionados aviones a traficantes de armas internacionales, lo cual frustró la renovación y la actualización de la flota aérea peruana (La Rosa, 2019).

Fernando Belaúnde Terry había adquirido veintiséis aviones y un simulador de vuelo durante su gobierno y en 1986, al año 
de gobierno, Alan García Pérez fue acusado por el entonces senador Carlos Malpica de haber recibido una coima por más de US\$ 100 millones por la compra frustrada y transferencia de aviones a terceros países (Malpica, 1993, p. iii).

Las consecuencias de esta situación fueron registradas en 1995, cuando Perú se enfrentó a Ecuador en el denominado “Conflicto del Cenepa” (Hernández, 1997).

b. El desmantelamiento de las capacidades operativas de la empresa militar Servicios Industriales de la Marina (SIMA) de la Marina de Guerra del Perú y de la Marina Mercante del Perú, lo cual provocó una crisis severa que se amplió hasta el gobierno de Ollanta Humala, en el cual se reactivó dicha empresa nacional para volver a ejecutar actividades industriales autónomas.

c. La estafa generada contra el gobierno Chino, con el empleo de tanques chinos en un desfile por fiestas patrias, luego de acordar comprar un lote de cien tanques chinos, tipo MBT-2000.

Ante el desistimiento en la compra de los mencionados tanques, el gobierno Chino accedió a una compensación económica promovida por García Pérez, pero el impacto en el Ejército peruano fue la desvinculación de la cooperación de dicho país.

Inclusive, el financiamiento para la ejecución de la adquisición de material bélico había sido aprobado, pero la manipulación que ejercía el partido político APra en el Poder Judicial y el Ministerio Público evitó que se ejecutara cualquier investigación sobre el particular (Mella, 2012).

2. Durante el gobierno de Alberto Fujimori, luego de la Guerra del Cenepa se optó por renovar la capacidad operativa de la Fuerza Aérea del Perú y se ejecutó la compra de aviones Mig-29 y Su-25 a Bielorrusia. 
La promulgación del Decreto de Urgencia N. o 029-1996 permitió la transferencia de US\$252 millones por 18 aviones Mig-29 y US\$ 150 millones por 14 aviones Su-25 (Velarde, 2001).

Sin embargo, la adquisición fue promovida por Vladimiro Montesinos y Alberto Fujimori con el objeto de adquirir material bélico de segunda mano y sin la capacidad bélica presentada a la opinión pública, motivo por el cual posteriormente se generó la necesidad de adquirir aviones y motores a Rusia, ante el registro de material bélico limitado (Arias et al., 2001, p. 84).

En forma complementaria, los gobiernos de Alejandro Toledo, Pedro Pablo Kuczynski y Martín Vizcarra Cornejo no registran actos de gobierno en procura de la mejora de la capacidad operativa de las Fuerzas Armadas, ni a nivel disuasivo, conforme las reglas de una guerra tradicional, ni tampoco se han adaptado a las nuevas necesidades del país, incluyendo la lucha contra el terrorismo y la lucha contra el narcotráfico.

Ollanta Humala Tasso, por su formación militar, estableció una política que ha procurado recuperar la capacidad defensiva de las Fuerzas Armadas peruanas (Infodefensa, 2019), que a la fecha ha permitido a la Marina de Guerra del Perú, a la Fuerza Aérea del Perú y al Ejército peruano tener una capacidad disuasiva significativa, pero que en términos generales sigue siendo limitada frente al potencial bélico de Brasil, Chile y Colombia, los países fronterizos.

Esta referencia permite detallar un panorama complicado respecto de la evaluación de las capacidades militares de las Fuerzas Armadas peruanas, porque en esencia se habían registrado dos etapas durante los años 1980-2000 y 2000-2020, con tres problemas de carácter significativo:

1. Las acciones de defensa de la integridad del territorio nacional respecto de las incursiones de Ecuador en la frontera norte del país, que provocaron los conflictos armados de los años 1982 (Conflicto del Falso Paquisha) y 1995 (conflicto del Cenepa), con un margen internacional positivo para Perú (Ferrero, 2018). 
2. La lucha contra el terrorismo, especialmente en contra de Sendero Luminoso, el movimiento Revolucionario Túpac Amaru (Sansó, 2017, p. 148) y el comando Rodrigo Franco de vinculación aprista (Cano, 2016).

3. La atención de desastres naturales, en particular en las acciones de apoyo cívico a las poblaciones que habían sido afectadas por desastres naturales como el fenómeno de El Niño, terremotos, inundaciones y obstrucción de caminos y carreteras.

En términos de capacidad operativa militar, Ollanta Humala observó esta situación y procedió a ejecutar las adquisiciones de aviones de transporte militar Spartan de Italia (Infodefensa, 2018) y la construcción de los buques multipropósito, BAP Pisco y BAP Paita (Gobierno del Perú, 2020a).

Durante la pandemia provocada por la Covid-19, el gobierno de Martín Vizcarra Cornejo adquirió dos aeronaves de transporte civil para la Fuerza Aérea del Perú y conforme el registro de las últimas adquisiciones, la capacidad operativa de la Fuerza Aérea ya era más cívica que militar.

Sin embargo, todo el conjunto de acciones vinculadas al ámbito de la defensa y la seguridad nacional cambió en el periodo comprendido entre octubre de 2019 y diciembre de 2020, principalmente por el registro de cuatro referencias que provocaron la reconfiguración de funciones de las Fuerzas Armadas del Perú. En este sentido:

1. La crisis de gobernabilidad del país entre octubre de 2019 y diciembre de 2020.

2. El control migratorio informal e ilegal de venezolanos por la frontera con Ecuador.

3. El apoyo social a la población durante la pandemia provocada por la Covid-19.

4. Patrulla y control de actividades en ultramar en las cercanías a la frontera marina peruana por parte de una flota de buques pesqueros chinos. 
Referencias que permiten evaluar cuatro ámbitos autónomos, pero que han incidido significativamente en la necesidad de adaptar la capacidad operativa de las Fuerzas Armadas peruanas.

\section{Las Fuerzas Armadas en el ámbito de la defensa de la gobernabilidad en la crisis peruana de noviembre del 2020}

La política nacional peruana ha estado en una crisis permanente entre abril de 2016 y diciembre de 2020 (Bermúdez-Tapia, 2020b, p. 343), especialmente porque se había registrado:

1. La victoria en las elecciones presidenciales de Pedro Pablo Kuczynski con un margen mínimo ante Keiko Fujimori, en dos procesos electorales sucesivos donde se había registrado el mayor ausentismo de la población. Esto generó una situación de deslegitimidad social en las instituciones políticas, lo cual permitió que el Congreso de la República tuviera el registro en mayoría absoluta del partido político que había perdido las elecciones presidenciales.

2. El constante enfrentamiento entre Kuczynski y la bancada parlamentaria del fujimorismo provocó dos procedimientos de vacancia por presunta incapacidad moral del presidente de la República. Esto generó que Kuczynski renunciara y la sucesión del vicepresidente al cargo de la presidencia de la República.

3. Un proceso de crisis política provocado porque Kuczynski otorgó un indulto humanitario a favor de Alberto Fujimori luego de haber pactado con el congresista Kenji Fujimori, hijo del expresidente y hermano de la rival política del presidente.

4. Un segundo momento de enfrentamiento político entre Martín Vizcarra y la bancada del fujimorismo, hecho que provocó la declaración de disolución del Congreso de la República el 30 de septiembre del 2019 por parte del poder ejecutivo, conforme una interpretación extensiva de la Constitución que fuese ratificada por el Tribunal Constitucional (2020). 
5. Un periodo de interregno parlamentario entre octubre de 2019 y el 15 de marzo del 2020 debido a la disolución del Congreso de la República y la instalación del nuevo Congreso de la República, de conformidad con lo establecido por el artículo $136 .^{\circ}$ de la Constitución Política de 1993.

6. Elecciones parlamentarias extraordinarias en enero de 2020 para elegir un nuevo Congreso que finalice el periodo presidencial del 2016-2021.

7. La declaratoria del estado de emergencia como consecuencia de la Covid-19, a través del Decreto Supremo N.o 184-2020-PCM, extendido por sucesivas ampliaciones de vigencia temporal.

8. La ejecución de dos procesos de vacancia presidencial a Martín Vizcarra por parte del nuevo Congreso del Perú.

9. La declaratoria de vacancia presidencial por incapacidad moral permanente, sin cumplir requisitos del debido proceso, como consecuencia de las acciones de Manuel Merino de Lama, presidente del Congreso de la República, que asumía el máximo cargo en el poder ejecutivo en el país debido a la renuncia de la segunda vicepresidenta Mercedes Araoz cuando se disolvió el Congreso de la República.

10. La crisis de los días 9 y 16 de noviembre de 2020, en la cual se registraron tres presidentes de la República (Martín Vizcarra, Manuel Merino y Francisco Sagasti) en cuatro procesos de sucesión presidencial, hecho que provocó la mayor situación de ingobernabilidad en el país desde la Guerra del Pacífico en 1879 (Bermúdez-Tapia, 2020c).

Conforme el registro de todos estos sucesos, las Fuerzas Armadas peruanas se presentaron como las entidades más sólidas ante la crisis política nacional, porque no aceptaron las presiones de los principales actores políticos que generaron la inestabilidad, de lo contrario se hubiera producido una crisis más aguda ("Vacancia presidencial...", 2020).

La subordinación a las autoridades civiles, sin importar su elección o legitimidad, fue una característica muy notoria en el mes de noviembre del 2020 y 
permite generar una eventual condición que determinó este comportamiento por parte de las Fuerzas Armadas peruanas:

1. El registro de una situación de fracaso total por parte de las organizaciones políticas que resultaba imposible de atender y solucionar en el plazo inmediato, con lo cual las Fuerzas Armadas optaron por no asumir una responsabilidad política.

2. La poca vinculación a un plan o proyección política de parte de las Fuerzas Armadas en la vida y el escenario político nacional, en particular porque las principales personalidades en los altos mandos militares no participaban en la vida política nacional, con excepción del héroe de guerra contra Ecuador, General Roberto Chiabra, quien ha focalizado su participación política a asuntos específicos en los ámbitos de la seguridad y la defensa nacional y educación pública.

3. El escaso margen de maniobrabilidad que podrían tener en el escenario nacional, tomando en cuenta el contexto de pandemia y la crisis político-social registrada ("Perú y el fracaso de la democracia sin instituciones", 2020).

\section{El apoyo social a la población durante la pandemia provocada por la Covid-19}

La declaratoria del estado de emergencia por parte del gobierno nacional ante el registro de la pandemia de la Covid-19 en Perú determinó que las Fuerzas Armadas peruanas ejecutaran acciones combinadas a favor de la población nacional, como:

1. Control preventivo de la población, en particular para desarrollar la inamovilidad social, la cual fue extrema durante el periodo comprendido entre el 16 y el 31 de marzo del 2020, primera etapa de la cuarentena sanitaria.

2. Control del tránsito y la movilidad humana durante toda la etapa de la pandemia: abril a diciembre de 2020, especialmente para limitar las aglomeraciones en la población.

3. Atención y suministro de alimentos, medicinas y apoyo logístico a los servicios sanitarios en todo el país, para lo cual se movilizó a casi 
todas las entidades y el personal en servicio activo y en reserva de las Fuerzas Armadas (Centro de Estudios Estratégicos del Perú, 2020).

4. Movilización de alimentos, medicinas y apoyo logístico a los servicios sanitarios a todo el territorio nacional, donde los vuelos humanitarios y la movilización de personal sanitario por parte de la Fuerza Aérea y la Policía Nacional fue una constante (Fuerza Aérea del Perú, 2020).

Por la dimensión de las circunstancias, se ejecutó un llamamiento extraordinario a la Reserva Orgánica de las Fuerzas Armadas peruanas, en particular a los licenciados en los años 2018, 2019 y febrero de 2020, con el Decreto Supremo N. ${ }^{\circ}$ 004-2020-DE (Gobierno del Perú, 2020b), con lo cual se generó una fuerza humana extraordinaria bajo la dirección de las autoridades militares y del gobierno nacional. Una situación nunca antes vista en el país que superó cualquier referencia bélica o situacional de crisis extrema.

En este sentido, corresponde detallar que las Fuerzas Armadas peruanas hasta antes de la pandemia de la Covid-19 habían registrado las siguientes situaciones, en función al problema en atención:

1. Durante la etapa del terrorismo, solo se habían empleado dependencias militares focalizadas en las zonas donde se registraba actividad terrorista y, por lo general, solo se empleaban recursos militares limitados.

Las bases militares ubicadas en Ayacucho, Huancavelica, Cusco y Huánuco eran esencialmente las empleadas para la lucha antisubversiva y por lo general se usaban tropas de escasa preparación militar.

3. Durante los enfrentamientos bélicos informales con Ecuador en 1982 y 1995, las Fuerzas Armadas peruanas solo emplearon los destacamentos militares ubicados en la zona de frontera norte (Chiabra, 2004).

Los destacamentos militares en Piura y Cajamarca fueron los únicos empleados y solo se dispuso un mínimo de material bélico para la ejecución de las actividades, especialmente porque la capacidad operativa de las aeronaves de la Fuerza Aérea era limitada. 
5. Durante la atención de emergencias sociales solo se empleaban los destacamentos militares ubicados en las zonas donde se registraban desastres naturales.

Por ello, la pandemia de la Covid-19 se puede considerar como la primera situación en la que las Fuerzas Armadas peruanas fueron empleadas con su total operatividad, en toda su historia nacional, por cuanto las circunstancias exigieron la movilidad de tropas, el destacamento de personal militar sanitario y la empleabilidad de casi todas las aeronaves y equipos bélicos de todas las Fuerzas Militares (Rasevic, 2021).

\section{El control migratorio informal e ilegal de venezolanos por la frontera con Ecuador}

Antes de la declaratoria del estado de emergencia provocada por la pandemia de la Covid-19, el gobierno nacional había modificado su política migratoria debido al volumen de ingreso de migrantes venezolanos por la frontera norte del país. El proceso migratorio venezolano se había incrementado durante los años 2018-2020 debido a las siguientes referencias:

1. El ingreso migratorio venezolano a Perú se había establecido sin condiciones, para lo cual se reguló una visa humanitaria (Consulado General del Perú en Caracas, 2020).

2. El ingreso, sin restricciones, al Seguro Integral de Salud, conforme a la Ley N. ${ }^{\circ}$ 29344, Ley Marco del Aseguramiento Universal en Salud.

3. El acceso a la educación pública y gratuita a todos los niños venezolanos, sin importar su condición migratoria o la de sus progenitores (Vadillo, 2018).

Sin embargo, durante la pandemia de la Covid-19, el ingreso de venezolanos de forma ilegal e indebida por la frontera nacional provocó la movilización de tropas y equipos bélicos en un nivel superior inclusive al empleado en los años 1982 y 1995, cuando se registraron hechos de naturaleza bélica ("Perú refuerza con blindados...”, 2020). 
El número de migrantes venezolanos ubicados en tránsito en Ecuador obligó al gobierno nacional a decretar una acción sin precedentes, en particular porque los efectos de la pandemia para los meses de abril y mayo del 2020 resultaban ser sumamente graves en el vecino país y se podía generar una situación sanitaria catastrófica en Perú.

\section{Patrulla y control de actividades en ultramar en las cercanías a la frontera marina peruana por parte de una flota de buques pesqueros chinos}

Una de las noticias que generó un impacto significativo en Sudamérica a finales del año 2020 fue el registro de una flota de buques chinos pescando de forma ilegal por las cercanías a Ecuador ("Armada del Ecuador detecta flota pesquera...”, 2020) y Argentina (Pesca Chubut, 2020), pese a que desde el 2017 se registraban estas actividades en las cercanías a la frontera marítima de Perú (“Perú refuerza con blindados...”, 2020).

El impacto significativo en lo económico y en lo que implica la protección de intereses de resguardo de la biósfera en la zona de soberanía marítima provocó que los países sudamericanos tuvieran una nueva perspectiva sobre la defensa de su soberanía y protección de intereses comerciales, de tal manera que debieron emplear a las fuerzas militares como única alternativa ante la dimensión del problema en ciernes.

Los Estados, en este sentido, han reconfigurado el alcance de las actividades de sus fuerzas militares en función de lo establecido por sus Constituciones, tal como hizo Perú, que justificó la ejecución de acciones de patrulla e interdicción de su Marina de Guerra en el extremo de las 200 millas de soberanía marítima para evitar que la flota china ingresara a una zona económica exclusiva nacional, en cumplimiento del artículo 67..$^{\circ}$ (Uso sostenible de recursos naturales) y artículo 68. (Conservación biológica y de las áreas naturales) de la Constitución Política de 1993.

Este nuevo reto para las Fuerzas Armadas peruanas en la política nacional sobre seguridad y defensa nacional constituye una referencia del modo en el cual las Fuerzas Militares deben estar preparadas para nuevos contextos internacionales (Sterner, 2007, p. 339), debido al cambio de conceptos tradicionales del uso de la guerra (Shiva, 2003). 
Consecuentemente, todo el contexto de la seguridad y la defensa nacional se debe adaptar a estas nuevas exigencias, lo cual genera la necesidad de adquirir equipos bélicos configurados para otras funciones (Espitia et al., 2020), mucho más idóneas para la vigilancia en amplios territorios como los desarrollados en el ámbito marítimo e inclusive el amazónico (Cardich, s. f.)

\section{Conclusiones}

Se están viviendo momentos históricos de cambios, especialmente en el ámbito del contexto internacional y sobre todo en las acciones de los países, en función de las nuevas necesidades que van surgiendo.

La provisión de recursos, de diferente naturaleza, como también la necesidad de una nueva geopolítica internacional han provocado cambios significativos en las políticas de los Estados, que deben hacer modificaciones estructurales en la administración de sus recursos, especialmente para garantizar su propia seguridad y defensa nacional.

Ante estas circunstancias, las fuerzas militares en Latinoamérica se han visto condicionadas a ejecutar nuevas funciones, por cuanto los intereses y la dinámica internacional se ha transformado. Esto resulta evidente en factores visibles como la ausencia de conflictos bélicos internacionales y la necesidad de reconfigurar las estructuras administrativas y bélicas de sus fuerzas militares.

En el Perú, este contexto se ha registrado de modo objetivo y las Fuerzas Armadas se encuentran en un proceso de adaptación a las nuevas exigencias del Estado, de la sociedad nacional y del futuro contexto geopolítico internacional, donde la protección del patrimonio y los intereses derivados del ejercicio soberano sobre el territorio constituyen una nueva prioridad.

Esta condición se aprecia en el impacto negativo que ha tenido la pandemia de la Covid-19, la cual ha demostrado la importancia superlativa de tener unas fuerzas militares operativas, especialmente para atender acciones que ordinariamente no son ejecutadas por ninguna entidad estatal debido a su alto requerimiento o a las condiciones extraordinarias de movilidad, logística o empleo de recursos humanos. 
En el proceso de cambio, las Fuerzas Armadas peruanas han visualizado un nuevo panorama y desde el gobierno de Ollanta Humala se ha planificado una política de adaptación a nuevas necesidades y requerimientos logísticos y bélicos. Este proceso ha permitido mejorar las capacidades operativas de todas las unidades de transporte de la Marina de Guerra del Perú, del Ejército peruano y de la Fuerza Aérea del Perú.

El nuevo contexto internacional y mundial es inminente, por ello otras fuerzas militares sudamericanas también han iniciado el proceso de transformación. Países como Colombia, Chile y Brasil se encuentran liderando este cambio de paradigmas en el empleo de las fuerzas militares en sus países.

\section{Referencias}

Alvarado Bedoya, O. (2014). El conflicto fronterizo entre Colombia y Nicaragua: Recuento histórico de una lucha por el territorio. Historia Caribe, 9(25), 241-271.

Álvarez Calderón, C. E., \& Duque Cruz, F. A. (2020). Oportunidades para las Fuerzas Militares de Colombia en operaciones multidimensionales de mantenimiento de paz. Revista Cientifica General José María Córdova, 18(29), 87-109. https://doi. org/10.21830/19006586.542

Arias Quincot, C., Avendaño Valdez, J., \& Vargas Llosa, M. (2001). Cómo Fujimori jodió al Perú. Milla Batres.

Armada del Ecuador detecta flota pesquera con 260 barcos en las cercanías de Galápagos. (2020, julio 16). El Universo. https://www.eluniverso.com/noticias/2020/07/16/nota/7908768/ armada-ecuador-detecta-flota-pesquera-260-barcos-cercanias

Basombrío Iglesias, C., \& Rospigliosi, F. (2006). La seguridad y sus instituciones en el Perú a inicios del siglo XXI: Reformas democráticas o militarismo. Instituto de Estudios Peruanos.

Bermúdez-Tapia, M. (2008). La Constitución a través de las sentencias del Tribunal Constitucional. Ediciones Legales.

Bermúdez-Tapia, M. (2020a). La influencia de la capacidad bélica sobre la soberanía nacional en las relaciones internacionales. Revista Cientifica General José María Córdova, 18(30), 291-306. http://dx.doi.org/10.21830/19006586.568

Bermúdez-Tapia, M. (2020b). El control parlamentario del Congreso del Perú a las acciones del ejecutivo en estados de emergencia en ocasión a una pandemia. Opinión Jurídica, 19(40), 341-367.

Bermúdez-Tapia, M. (2020c). El debido proceso en el procedimiento parlamentario de vacancia presidencial peruano. Revista da Faculdade de Direito de São Bernardo Do Campo, 26(2), 22. http://orcid.org/0000-0003-1576-9464 
Bruzzone, E. (2008). Las guerras del agua. América, el objetivo más codiciado. Capital Intelectual.

Cancillería Argentina. (2016, marzo). Argentina extiende su Plataforma Continental sobre Océano Atlántico. https://eviet.cancilleria.gob.ar/es/content/argentina-extiende-su-plataforma-continental-sobre-océano-atlántico

Cano, G. (2015). Comando Rodrigo Franco: ¿Leyenda urbana? Revista IDEELE, (258). https://revistaideele.com/ideele/revista/258

Cardich Pulgar, J. L. (s. f.). Modernización y transparencia en la gestión de las Fuerzas de Defensa. Fuerza Aérea del Perú. https://www.resdal.org/producciones-miembros/redes03-cardich.pdf

Cañedo Andalia, R. (2014). Un acercamiento a la evolución histórica y del estado actual de la actividad bibliológico-informacional. Editorial Universitaria.

Centro de Estudios Estratégicos del Ejército del Perú. (2020). La labor del Ejército del Perú en el contexto del Covid-19. Ejército del Perú. https://ceeep.mil.pe/wp-content/ uploads/2020/06/La-labor-del-Ejercito-Peruano-en-el-contexto-del-COVID-19-web.pdf

CEPAL. (2020). Amazonía posible y sostenible. CEPAL. https://www.cepal.org/sites/default/ files/news/files/folleto_amazonia_posible_y_sostenible.pdf

Chan, J. (2005). La china de Mao y la Guerra Fría. Paidós.

Chiabra León, R. (2004). Cenepa: Misión de honor. El fin de un siglo de conflictos. Format.

Consulado General del Perú en Caracas. (2020). Visa Humanitaria para venezolanos. http://www. consulado.pe/es/Caracas/tramite/Paginas/Visas/Visa-Humanitaria-para-Venezolanos.aspx

Cujabante Villamil, X. A. (2019). La disciplina de las relaciones internacionales en Brasil: Un análisis desde la sociología de la ciencia. Revista Cientifica General José María Córdova, 17(26), 289-305. https://doi.org/10.21830/19006586.417

De Pieri, V. (2013). Seguridad y defensa en Sudamérica: Entre la cooperación y el conflicto. Edudeba.

Dollfus, O. (2014). Territorios andinos: Reto y memoria. Instituto de Estudios Peruanos.

Espitia Cubillos, A. A., Agudelo Calderón, J. A., \& Buitrago Suescún, Ó. Y. (2020). Innovaciones tecnológicas en las fuerzas militares de los países del mundo: Una revisión preliminar. Revista Científica General José Maria Córdova, 18(29), 213-235. https:// doi.org/10.21830/19006586.537

Ferrero Costa, E. (2018). Perú-Ecuador: El proceso para lograr la paz. PUCP.

Fernández Menéndez, J. (2020). La nueva guerra. Del Chapo al fentanilo. Penguin Random House.

Fuerza Aérea del Perú. (2020, octubre). Operaciones aéreas durante la emergencia sanitaria Covid-19. https://www.fap.mil.pe/index.php/noticias-fap/covid-19

Fukuyama, F. (2008). Falling behind. Explaining the development gap between Latin America and the United States. Oxford University Press.

García Reyes, M., \& Ronquillo Jarillo, G. (2005). Estados Unidos, petróleo y geopolítica: las estrategias petroleras como un instrumento de reconfiguración geopolitica. Instituto Mexicano del Petróleo. 
García Suárez, A. L. (2019). El desarrollo de la democracia colombiana y sus efectos en el binomio Fuerzas Militares-ciudadanía. Revista Cientifica General José Maria Córdova, 17(26), 253-268. https://doi.org/10.21830/19006586.393

Gatt Corona, G. (2013). El derecho de guerra contemporáneo: Reflexiones desde el pensamiento de Francisco de Vitoria. ITESO.

Guerrero López, L. (2019). Participación de las Fuerzas Militares de Colombia en operaciones de mantenimiento de paz 2019-2023. Revista Cientifica General José María Córdova, 17(25), 5-24. https://doi.org/10.21830/19006586.355

Giraldo Chaparro, F. R. (2019). Las Fuerzas Militares como instrumento de socialización del Estado. Revista Científica General José María Córdova, 17(28), 939-970. https://doi. org/10.21830/19006586.517

Gobierno del Perú. (2017). Decreto Supremo N.o 012-2017-DE. Decreto Supremo que aprueba la Política de Seguridad y Defensa Nacional. El Peruano. https://busquedas.elperuano. pe/normaslegales/decreto-supremo-que-aprueba-la-politica-de-seguridad-y-defen-decreto-supremo-n-012-2017-de-1600032-1/

Gobierno del Perú. (2020a). Decreto Supremo de llamamiento extraordinario de la reserva orgánica perteneciente a las últimas clases, licenciados en los ańos 2018, 2019 y febrero 2020. El Peruano. https://busquedas.elperuano.pe/normaslegales/decreto-supremo-de-llamamiento-extraordinario-de-la-reserva-decreto-supremo-no-004-2020-de-1865180-3/

Gobierno del Perú. (2020b, febrero 17). Industria naval del Perú se ha convertido en referente en Latinoamérica. https:/www.gob.pe/institucion/pcm/noticias/82338-industria-naval-del-peru-se-ha-convertido-en-referente-en-latinoamerica

Griffiths Spielman, J. (2011). Teoría de la seguridad y defensa en el continente americano. Análisis de los casos de EE.UU. de América, Perú y Chile. RIL Editores.

Hernández, L. (1997). La guerra del Cenepa: diario de un comandante. Corporación Editora Nacional.

Hurtado Noriega, C., \& Doria Velarde, A. J. (2020). Nuevos roles de las fuerzas armadas en seguridad. Experiencias de México, Colombia y Perú. Revista Cientifica General José María Córdova, 18(30), 379-398. https://doi.org/10.21830/19006586.548

Infodefensa.com. (2018, noviembre 17). Perú comprará otros dos Spartan y será su mayor operador en Latam. https://www.infodefensa.com/latam/2018/11/17/noticia-comprara-otros-spartan-mayor-operador-latam.html

Infodefensa.com. (2019, septiembre 6). Perú: 2,070 millones de dólares en cinco años para mejorar sus FFAA. https://www.infodefensa.com/latam/2016/09/06/opinion-millones-dolares-cinco-mejorar.php

Infodefensa.com. (2020, marzo 20). La FACh detecta una nueva fuga de pilotos al mercado civil. https://www.infodefensa.com/latam/2018/03/20/noticia-detecta-nueva-pilotos-mercado-civil.html

Infodron.es. (2020, abril 20). Argentina, Brasil, Chile y Colombia avanzan en la legislación sobre drones. http://infodron.es/id/2018/04/20/noticia-argentina-brasil-chile-colombia-avanzan-legislacion-sobre-drones.html 
La Rosa Vásquez, R. (2019, mayo 6). Alan García: una vida política marcada por la polémica. El Comercio. https://elcomercio.pe/politica/alan-garcia-vida-politica-marcada-polemica-noticia-632459-noticia/

López Escarcena, S. (2014). La disputa marítima entre Perú y Chile: Comentario de la sentencia de la Corte Internacional de Justicia, de fecha 27 de enero de 2014. Revista Chilena de Derecho, 41(3), 1133-1153. https://dx.doi.org/10.4067/S0718-34372014000300014

Malpica Silva Santisteban, C.(1993). Pájaros de alto vuelo: Alan García, el BCCI y los Mirage. Inca.

Mella, R. (2012). "Fue una vergüenza”. El caso del desfile de los tanques chinos. IDL Reporteros. https://www.idl-reporteros.pe/fue-una-verguenza/

Moreno Mancera, J. D. (2014). Relaciones cívico-militares en Colombia: Supremacía y control de los partidos políticos sobre la organización militar. Revista Cientifica General José María Córdova, 12(13), 333-352. https://doi.org/10.21830/19006586.166

Morote Costa, F. (1999). Después de la Guerra Fría y otros articulos: Artículos y conferencias de historia y política contemporáneas. Fundamentos.

Narváez, Z., \& Rodríguez M. (2005). Expansionismo económico y militar de Estados Unidos en América Latina y el Caribe. Centro de Estudios Internacionales. http://biblioteca. clacso.edu.ar/Nicaragua/cei/20120809024822/expansionismo.pdf

Pastrana Buelvas, E., \& Gehring, H. (2018). La problemática del tráfico ilícito de drogas: Impactos regionales y globales. Editorial Pontificia Universidad Javeriana / Konrad Adenauer Stiffung.

Perú refuerza con blindados el control de frontera con Ecuador por el coronavirus. (2020, abril 10). Gestión. https://gestion.pe/peru/peru-refuerza-con-blindados-el-control-de-frontera-con-ecuador-por-el-coronavirus-noticia/

Perú y el fracaso de la democracia sin instituciones. (2020, noviembre 16). DW. https://www. dw.com/es/perú-y-el-fracaso-de-la-democracia-sin-instituciones/a-55619454

Pesca Chubut. (2020, junio 15). Buques chinos navegan por el Estrecho de Magallanes luego de posiblemente estar pescando ilegalmente en el mar argentino. https://pescachubut.com/ buques-chinos-navegan-por-el-estrecho-de-magallanes-luego-de-posiblemente-estar-pescando-ilegalmente-en-el-mar-argentino/

Rasevic, Z. (2021). El recurso a la fuerza militar en la emergencia sanitaria de Covid-19: Una justificación. Revista Cientifica General José María Córdova, 19(35). https://doi. org/10.21830/19006586.777

Roland, A. (2016). War and technology. A very short introduction. Oxford University Press.

Sansó, D., \& Pascual, R. (2017). Democracias bajo presión. Dykinson.

Shaw, M. (2005). The new Western way of war: Risk-transfer war and its crisis in Iraq. Polity. Shiva, V. (2003). Las guerras del agua: Privatización, contaminación, lucro. Siglo XXI.

Sierra-Zamora, P. A., Aponte, S. G. S., Fernández-Osorio, A. E., \& Ortiz, T. L. F. (2020). El control de cultivos ilícitos en zonas donde se han registrado desplazamientos forzados. En Sierra-Zamora, P. A., Bermúdez Tapia, \& Arango Calderón, I. Y., Elementos judiciales y procesales en contextos de cambios sociales (pp. 20-52). Sello Editorial ESMIC. https:// doi.org/10.21830/9789585284883.01 
Sierra-Zamora, P. A., Bermúdez-Tapia, M., \& Fernández-Osorio, A. E. (2020). Las diferencias entre Colombia y Perú en la finalización del conflicto armado interno. En M. Bermúdez-Tapia, P. A. Sierra-Zamora \& A. E. Fernández-Osorio (eds.), El Estado ante emergencias sociales (pp. 85-100). Sello Editorial ESMIC. https://doi. org/10.21830/9789585284869.04

SIN: "Hay más de 400 barcos asiáticos que depredan la pota en mar peruano". (2017, abril 9). Gestión. https://gestion.pe/economia/sni-hay-400-barcos-asiaticos-depredan-pota-mar-peruano-132771-noticia/

Sterner, T. (2007). Instrumentos de politica económica para el manejo del ambiente y los recursos naturales. Banco Mundial.

Tansey, G., \& Rajotte, T. (2009). El control futuro de los alimentos: Guía de las negociaciones y reglas internacionales sobre la propiedad intelectual, la biodiversidad y la seguridad alimentaria. Mundi-Prensa.

Trebilcock, M., \& Mota Prado, M. (2011). What makes poor countries poor? Institucional determinants of development. Edward Elgar.

Tribunal Constitucional. (2020). Expediente N. o 0011-2019-PI. Caso de la participación de las Fuerza Armadas en la interdicción del Tráfico Ilícito de Drogas en zonas declaradas en estado de emergencia. https://tc.gob.pe/jurisprudencia/2020/00011-2019-AI.pdf

Tribunal Constitucional. (2019). Expediente N. ${ }^{\circ}$ 0006-2019-CC/TC. Sentencia del caso sobre la disolución del Congreso de la República. https://tc.gob.pe/jurisprudencia/2020/00006-2019-CC.pdf

Vacancia presidencial: Manuel Merino reconoce que llamó a oficial de las Fuerzas Armadas y dice que fue un "llamado a la calma". (2020). RPP. https://rpp.pe/politica/gobierno/vacanciapresidencial-manuel-merino-reconoce-que-llamo-a-oficial-de-las-fuerzas-armadas-y-diceque-fue-un-llamado-a-la-calma-noticia-1292157

Vadillo Vila, J. (2018, septiembre 13). Niños venezolanos en Perú, su integración es una tarea pendiente. Andina. https://andina.pe/agencia/noticia-ninos-venezolanos-peru-su-integracion-es-una-tarea-pendiente-725049.aspx

Varea, A. (1995). Marea Negra en la Amazonía. Conflictos socioambientales vinculados a la actividad petrolera en el Ecuador. Instituto Latinoamericano de Investigación.

Vasapollo, L., Petras, J., \& Casado, M. (2007). Potencias en conflicto: La pugna por la hegemonía mundial. El Viejo Topo.

Velarde, C. (2001, febrero 26). Fujimori compró aviones de segunda mano y los pagó como nuevos. Estafa a la seguridad nacional. La República. https://larepublica.pe/politica/332416-fujimori-compro-aviones-de-segunda-mano-y-los-pago-como-nuevos-estafa-a-la-seguridad-nacional/

Velázquez Gutiérrez, M. (2014). El discurso hegemónico ambiental a través de organismos de cooperación y su influencia en las relaciones internacionales. Revista Cientifica General José María Córdova, 12(13), 191-202. https://doi.org/10.21830/19006586.160

Welzer, H., \& Obermeier, A. (2010). Guerras climáticas: Por qué nos mataremos (y nos matarán) en el siglo XXI. Kats. 


\section{Anexo}

\section{Artículos de la Constitución Política del Perú de 1993}

Artículo 8. ${ }^{\circ}$. Represión al Tráfico Ilícito de Drogas. El Estado combate y sanciona el tráfico ilícito de drogas. Asimismo, regula el uso de los tóxicos sociales.

Artículo 43. ${ }^{\circ}$. Estado democrático de derecho. Forma de Gobierno de la República del Perú es democrática, social, independiente y soberana. // El Estado es uno e indivisible. // Su gobierno es unitario, representativo y descentralizado, y se organiza según el principio de la separación de poderes.

Articulo 118. ${ }^{\circ}$. Atribuciones del Presidente de la República. Corresponde al Presidente de la República: [...] 4. Velar por el orden interno y la seguridad exterior de la República. [...]. 14. Presidir el Sistema de Defensa Nacional; y organizar, distribuir y disponer el empleo de las Fuerzas Armadas y de la Policía Nacional.

Artículo 137. ${ }^{\circ}$. Estados de excepción. Estado de emergencia y estado de sitio. El Presidente de la República, con acuerdo del Consejo de Ministros, puede decretar, por plazo determinado, en todo el territorio nacional, o en parte de él, y dando cuenta al Congreso o a la Comisión Permanente, los estados de excepción que en este artículo se contemplan:

1. Estado de emergencia, en caso de perturbación de la paz o del orden interno, de catástrofe o de graves circunstancias que afecten la vida de la Nación. En esta eventualidad, puede restringirse o suspenderse el ejercicio de los derechos constitucionales relativos a la libertad y la seguridad personales, la inviolabilidad del domicilio, y la libertad de reunión y de tránsito en el territorio comprendidos en los incisos 9, 11 y 12 del artículo 2 y en el inciso 24, apartado f del mismo artículo. En ninguna circunstancia se puede desterrar a nadie. El plazo del estado de emergencia no excede de sesenta días. Su prórroga requiere nuevo decreto. En estado de emergencia las Fuerzas Armadas asumen el control del orden interno si así lo dispone el Presidente de la República.

3. Estado de sitio, en caso de invasión, guerra exterior, guerra civil o peligro inminente de que se produzcan, con mención de los dere- 
chos fundamentales cuyo ejercicio no se restringe o suspende. El plazo correspondiente no excede de cuarenta y cinco días. Al decretarse el estado de sitio, el Congreso se reúne de pleno derecho. La prórroga requiere aprobación del Congreso.

Artículo $164 .^{\circ}$. Dirección, preparación y ejercicio del Sistema de Defensa Nacional. La dirección, la preparación y el ejercicio de la Defensa Nacional se realizan a través de un sistema cuya organización y cuyas funciones determina la ley. El Presidente de la República dirige el Sistema de Defensa Nacional. // La ley determina los alcances y los procedimientos de la movilización para los efectos de la defensa nacional.

Artículo 165.․ Finalidad de las Fuerzas Armadas. Las Fuerzas Armadas están constituidas por el Ejército, la Marina de Guerra y la Fuerza Aérea. Tienen como finalidad primordial garantizar la independencia, la soberanía y la integridad territorial de la República. Asumen el control del orden interno de conformidad con el artículo 137. ${ }^{\circ}$ de la Constitución.

Artículo 166. ${ }^{\circ}$. Finalidad de la Policía Nacional. La Policía Nacional tiene por finalidad fundamental garantizar, mantener y restablecer el orden interno. Presta protección y ayuda a las personas y a la comunidad. Garantiza el cumplimiento de las leyes y la seguridad del patrimonio público y del privado. Previene, investiga y combate la delincuencia. Vigila y controla las fronteras.

Artículo 167.․ Jefe Supremo de las Fuerzas Armadas y Policía Nacional. El Presidente de la República es el Jefe Supremo de las Fuerzas Armadas y de la Policía Nacional. 